\title{
MAINTAINING A REPUTATION AGAINST A LONG-LIVED OPPONENT
}

\author{
By Marco Celentani, Drew Fudenberg, David K. Levine, and \\ WOLFGang PESENDORfer ${ }^{1}$
}

\section{INTRODUCTION}

WE CONSIDER A GAME between a patient player 1 and a nonmyopic but less patient opponent, player 2. As usual in reputation models, we suppose that the patient player's type is private information, and that he may be a "commitment type" who is locked into playing a particular strategy. We investigate the extent to which an uncommitted or "normal" type of patient player can exploit his less patient opponent's uncertainty to maintain a reputation for playing like a commitment type.

Most previous work on reputation effects has supposed that player 2 is in fact completely myopic, or equivalently that player 2 corresponds to a sequence of short run players (see Kreps and Wilson (1982), Milgrom and Roberts (1982), Fudenberg and Levine $(1989,1992)){ }^{2}$ Since a myopic player 2 will play a short-run best response in each period to that period's expected play, the best possible commitment for the long run player is to the Stackelberg strategy for the corresponding static game.

This paper considers the case in which the patient player, player 1 , has discount factor $\delta_{1}$ near 1 , while his opponent, player 2 , is an infinite-lived player who discounts future payoffs with a smaller discount factor $\delta_{2}$. Perhaps the best way to interpret this assumption of unequal discount factors is to interpret the model as a shorthand for a situation where player 1 faces a large number of identical player 2's, each of whom observe all previous play. Under one interpretation the player 2's alternate play so that, for example, the first player 2 moves in periods $1, N+1$, etc. Alternatively, the first player 2 might move in periods 1 to $N$, the second in $N+1$ to $2 N$, and so on. Under either interpretation, the key is that player 1 cares more about future payoffs of this game than player 2 does, because he will be playing in more future periods. ${ }^{3}$

A game with a nonmyopic opponent differs from one with a myopic opponent in two main ways. First, because a nonmyopic opponent cares about future payoffs, the static Stackelberg strategy is no longer necessarily the best possible commitment: Higher payoffs can sometimes be attained by the use of rewards and punishments. In the prisoner's dilemma, for example, "tit-for-tat" is a better commitment against a nonmyopic opponent than the Stackelberg strategy of defecting. Second, it may be difficult to demonstrate that one is using a strategy with rewards and punishments unless these rewards and punishments are occasionally carried out. This is similar to the way in which

\footnotetext{
${ }^{1}$ The authors are grateful for financial support from NSF Grants SBR-9223320, SBR-9223175, SBR-9409180, DGICYT PB92-0245, Human Capital Mobility Programme ERBCHBICT940975, and the UCLA Academic Senate.

${ }^{2}$ Celentani and Pesendorfer (1995) consider dynamic games with one large player and a continuum of small but long lived players. Small players in this setting care about the future but cannot influence the relevant history of the game and hence are strategically myopic.

${ }^{3}$ Thus the situation is similar to the "sequential contests" model of Fudenberg and Kreps (1987).
} 
incorrect off-path beliefs can weaken reputation effects in the play of extensive-form games against myopic opponents (Fudenberg and Levine (1989)). ${ }^{4}$

Our main assumption is that player 1 does not observe player 2's intended action, but only sees an imperfect signal of it, as in a model of moral hazard. We assume that the support of the distribution of signals is independent of how player 2 plays. Intuitively, this ensures that every reward and punishment in player 1's strategy will occasionally be triggered, so that player 2 will learn how player 1 responds to all sequences of public outcomes. As a result, player 1's equilibrium payoff is bounded below by what he could get through commitment in the repeated game. In particular, if player 2 is sufficiently patient, player 1 gets approximately the greatest feasible payoff consistent with individual rationality of player 2 .

This conclusion holds with an arbitrarily small amount of noise. However, as the amount of noise shrinks, the patient player's discount factor must be increasingly close to one to ensure that its Nash equilibrium payoff is close to its limit value. Consequently, our result is of the most relevance when the amount of noise is "significant."

The first general study of reputation with two nonmyopic players is Schmidt (1993), who studied perfect observability. He showed that the long-run player can guarantee at least the payoff he would get from precommitment to a static strategy that minmaxes his opponent. This is a good bound in some games, but in others, such as the prisoner's dilemma, it imposes no restrictions beyond those implied by individual rationality. Subsequently Cripps, Schmidt, and Thomas (1996) provided tight bounds in the case of perfect observability; Cripps and Thomas (1994) provided the parallel result when both players have time-average payoffs. In each of these papers, reputation effects need not allow the patient player to obtain as high a payoff as he could were he able to publicly commit himself to follow any strategy of his choice.

The key point is that with perfectly observed actions, the problem of off-path beliefs can prevent player 1 from obtaining the payoff he would most prefer. Schmidt (1993) gives an example of a perfect Bayesian equilibrium in which player 2's inability to learn the strategy played off the equilibrium path prevents player 1 from achieving the payoff he would get with a public commitment. This example is based on the presence of a "perverse" type who plays like the "good" commitment type on the equilibrium path, but responds to deviations in a history dependent way. Cripps, Schmidt, and Thomas (1996) show that the perverse type is not required if we consider only Nash equilibria. Their Theorem 2 applies to games with observed actions where the patient player is either "normal" or plays an arbitrary finitely-complex strategy. It shows that there is a Nash equilibrium where player 1's payoff is not substantially above the most he could obtain by playing a constant action, with player 2 choosing the individually rational response to this action that player 1 likes least.

In Section 5 we provide an example that shows the assumption of imperfect observability can in general not be dispensed with, even if we restrict to perfect Bayesian equilibria. The example shows that with perfect observability of actions there are perfect Bayesian equilibria of the infinitely repeated game in which player 1's payoff is bounded away from the Stackelberg payoff.

Finally, we should acknowledge that Aoyagi (1994) independently obtains a result similar to ours for the case where player 1 maximizes his time-average payoff while player

\footnotetext{
${ }^{4}$ Celentani (1996) uses multiple types of short-run players to get around the problem of unobservable off-path behavior of the long-run player in repeated games with observable actions. His approach can be extended to short run players who live for more than one period.
} 


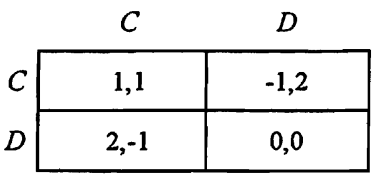

FIGURE 1

2 discounts. Aoyagi's paper differs in interpreting the noise as "trembles," and, more significantly, in considering a complex class of "commitment types" that may be empty in some games, but the basic intuition for his results is the same.

To illustrate an application of our Theorems, as well as how they differ from the bounds of Cripps, Schmidt, and Thomas (1996), we examine a version of the Prisoner's Dilemma. It should be noted that the bound developed by Schmidt (1993) and by Cripps, Schmidt, and Thomas (1996) does not imply any restriction on the Nash equilibria of this game: the best player 1 can get while minmaxing player 2 is his own minmax. We start with a traditional prisoner's dilemma (see Figure 1). We add incomplete observability, by supposing that if a player chooses a particular action then there is a small chance that the realized action will be different. In particular suppose that conditional on choosing action $C$ (or $D$ ) the realized action will be $C(D)$ with probability $1-\varepsilon$ and $D(C)$ with probability $\varepsilon$. For this example a simple calculation shows that the greatest socially feasible payoff for player 1 that gives player 2 at least the minmax is $3 / 2-2 \varepsilon$. Our results imply that if player 2 is patient, then a more patient player 1 will receive a payoff close to $3 / 2-2 \varepsilon$ in every Nash equilibrium of this prisoner's dilemma with commitment types. $^{5}$

\section{THE MODEL}

Let $g$ denote a stage game between two players, player 1 (the patient player) and player 2 . We denote by $A_{1}, A_{2}$ the finite (pure) action sets of the two players in the stage game with generic elements $a_{1}, a_{2}$, and use $\alpha_{1} \in \tilde{A_{1}}, \alpha_{2} \in \tilde{A_{2}}$ for mixed actions. We denote by $A, \tilde{A}$ the corresponding spaces of profiles. At the end of the stage game both players observe a stochastic outcome drawn from a finite set, $y \in Y$, but players do not observe each other's action. The probability distribution over outcomes depends on the action profile and is given as $\rho(\cdot \mid a)$; for mixed actions $\rho(\cdot \mid \alpha)$ is defined in the obvious way. Stage game payoffs, denoted $u_{i}(\alpha)$, take the form $u_{i}(a) \equiv \Sigma_{y} u_{i}\left(a_{i}, y\right) \rho(y \mid a)$ and therefore the realized payoffs do not convey additional information about the other player's action.

We consider an infinitely repeated game, $G^{\infty}$, constructed from $g$ as follows. The public history at date $t, h^{t}$, is the sequence of past realizations of the outcome $y$. Player $i$ 's private history at $t, h_{i}^{t}$, is the sequence of actions $i$ chose in previous periods. Let $H$, $H_{1}$, and $H_{2}$ denote the sets of infinite public and private histories, for player 1 and 2 respectively.

Player 1 can be one of countably many types, $\omega \in \Omega$. These types are drawn from a common knowledge prior $\mu$ assigning positive probability to all points in $\Omega$. Player 1 's

\footnotetext{
${ }^{5}$ Note that this payoff can be achieved if player 2 always chooses $C$ and player 1 alternates between $C$ and $D$.
} 
type is private information. We focus on a particular type $\omega_{0} \in \Omega$, which we refer to as the normal type. Type $\omega_{0}$ seeks to maximize the average present value of $u_{1}$ using the discount factor $\delta_{1}$, while player 2 maximizes the average present value of $u_{2}$ with the discount factor $\delta_{2}$. Types of player 1 other than type $\omega_{0}$ have von Neumann-Morgenstern preferences over sequences of own actions and public outcomes, but these are not necessarily representable in a time separable form.

A behavior strategy for a type of player 1 or a behavior strategy for player 2 specifies a time indexed sequence of maps from private (for that player) and public histories to mixed actions (for that player). We denote these by $\sigma_{1}$ and $\sigma_{2}$ respectively. We also define $u_{i}^{t}\left(\sigma_{1}, \sigma_{2}\right)$ to be the corresponding period $t$ expected payoff. Finally, a behavior strategy for player $1, \jmath_{1}$, specifies a behavior strategy for each type. A Nash equilibrium is a behavior strategy for each player such that given the opponent's behavior strategy, no other behavior strategy yields a distribution over time sequences of own actions and public outcomes that is preferred to that in the proposed equilibrium. It is quite easy to show by taking limits of finite truncations of this infinite game that Nash equilibria exist. ${ }^{6}$ Let $E_{1}\left(\delta_{1}, \delta_{2}\right)$ denote the least (inf) expected payoff to player 1 conditional on type $\omega_{0}$ in any Nash equilibrium.

Say that a behavior strategy for player 1 has bounded recall if there exists a number $N$ such that play at time $t$ is entirely determined by the history between $t-N$ and $t-1$. A type of player 1 whose preferences make the type behavior strategy $\sigma_{1}$ strictly dominant is called committed to that strategy, and we write the type as $\omega\left(\sigma_{1}\right)$.

We make four key assumptions:

ASSUMPTION 1: If $\sigma_{1}$ is a pure strategy of bounded recall, then $\omega\left(\sigma_{1}\right) \in \Omega$, that is, it has positive probability.

AsSUMPTION 2: If $\rho\left(\cdot \mid \alpha_{1}, \alpha_{2}\right)=\rho\left(\cdot \mid \alpha_{1}^{\prime}, \alpha_{2}\right)$, then $\alpha_{1}=\alpha_{1}^{\prime}$.

AssumPtion 3: The support of $\rho(\cdot \mid \alpha)$ is independent of $\alpha_{2}$.

Define $\underline{u}_{2} \equiv \min _{a_{1}} \max _{a_{2}} u_{2}(a)$ to be the (pure strategy) minmax for player 2 .

AssumPtion 4: There exists a pure profile a such that $u_{2}(a)>\underline{u}_{2}$.

Assumption 1 ensures there are enough irrational types. (Since the set of bounded-recall strategies is countable this is consistent with our restrictions to a countable number of types.) Assumption 2 requires that regardless of the play of player 2 the information revealed by the outcomes identifies the action of player 1 . If it fails, player 2 may play an action that precludes him from learning what stage-game action player 1 is playing, preventing player 1 from developing a reputation, even when player 2 is myopic. Note that the assumption is satisfied if player 1's actions are perfectly observed, as in the previous papers on reputation effects with two long-run players.

Assumption 3 is the substantive assumption: It says that player 2 cannot determine the set of possible outcomes through his own action. Note that this assumption does not require that player 1's action be imperfectly observed and indeed, whether player 1's action is observed or not is irrelevant.

\footnotetext{
${ }^{6}$ See, for example, Fudenberg and Levine (1983).
} 
Assumption 4 says that there is a profile that is better for player 2 than the pure strategy minmax payoff. If we used mixed strategies in place of pure, this would be a mild nondegeneracy condition: failure would mean that the indifference of player 2 might well make him immune to threats by player 1 . We restrict attention to the pure strategy minmax in order to avoid the complications involved in maintaining a reputation for playing a mixed strategy. ${ }^{7}$ The existence of a profile better for player 2 than the pure strategy minmax rules out some interesting games, but the assumption is satisfied in other games of interest, such as the prisoner's dilemma and the battle of the sexes.

Let $G_{0}^{N}$ be the complete-information game corresponding to the $N$ period repetition of $g$; players discount their payoffs in $G_{0}^{N}$ using the same discount factors $\delta_{1}$ and $\delta_{2}$ as in the infinity repeated game. ${ }^{8}$ Let $G^{N}$ be the $N$ period game with discount factors $\delta_{1}$ and $\delta_{2}$, type space $\Omega$, and prior $\mu$.

Before analyzing reputation in our model, we calculate as a benchmark how much player 1 might hope to get by precommitting. First we define a set of payoffs for player 1 .

Definition 1: $\nu_{1} \in V_{1}\left(\delta_{2}\right)$ if and only if for every $\varepsilon>0$ there is an $N$ and a pure strategy $\sigma_{1}^{N}$ such that for every best response $\sigma_{2}^{N}$ to $\sigma_{1}^{N}$ in $G_{0}^{N},(1 / N) \sum_{t=1}^{N} u_{1}^{t}\left(\sigma_{1}^{N}, \sigma_{2}^{N}\right)$ $\geq \nu_{1}-\varepsilon$.

Through a public precommitment (to a pure strategy) a patient player can guarantee himself $\bar{\nu}_{1}\left(\delta_{2}\right) \equiv \sup V_{1}\left(\delta_{2}\right){ }^{9}$

\section{AN IMPATIENT PLAYER 2}

Our main result is:

THeOREM 1: Suppose that Assumptions 1-3 hold. Then $\liminf \operatorname{in}_{\delta_{1} \rightarrow 1} E_{1}\left(\delta_{1}, \delta_{2}\right) \geq \bar{\nu}_{1}\left(\delta_{2}\right)$.

In other words, if player 1 is very patient, then he gets nearly as much in any Nash equilibrium as the greatest amount consistent with player 2 choosing a best response in a finite truncation of the game.

The idea is that if player 1 commits to an appropriate bounded recall strategy and player 2 plays a best response to it, then player 1 gets a payoff very close to the lower bound given above. Note that since the strategy has bounded recall there is a type who is committed to playing this strategy. In the usual reputational story, this would mean that if player 1 chooses this strategy, player 2 must either play a best response to it, or come

\footnotetext{
${ }^{7}$ These complications were addressed in the context of a myopic player 2 in Fudenberg and Levine (1992). The restriction is largely a matter of technical convenience: for player 1 to develop a reputation for mixed strategy punishments it would be necessary to allow a continuum of types. Working with a continuum of types increases the complexity of the notation substantially.

${ }^{8}$ To reduce notation we do not track this dependence explicitly in the notation. Since we do not need to discuss the $N$-period game with time-average payoffs, no confusion should result.

${ }^{9}$ Because we restrict attention to pure strategy commitment types, the worst punishment that player 1 can hope to "teach" player 2 to fear is the pure-strategy minmax; this restriction on punishments can result in a lower maximum payoff for player 1 than if mixed punishments were considered.
} 
to believe that he faces a committed type. The situation here is complicated by the need to show that player 2 can learn the punishment strategy of player 1 without deviating: this is where Assumption 3 comes in.

We proceed via several Lemmas. Our initial focus is on the response of player 2 to bounded recall strategies in $G_{0}^{N}$.

LeMMA 1: For every $\eta>0$ there exists an $N, \underline{\delta}_{1}<1, \varepsilon>0$ and pure strategy $\sigma_{1}^{N}$ for player 1 in $G_{0}^{N}$, such that if $1>\delta_{1} \geq \underline{\delta}_{1}$ and player 2 plays an $\varepsilon$-best response to $\sigma_{1}^{N}$ in $G_{0}^{N}$, then the payoff to player 1 is at least $\bar{\nu}_{1}\left(\delta_{2}\right)-\eta$.

Proof: Omitted; see Celentani et al. (1993).

If $\sigma^{N}$ is a profile in $G_{0}^{N}$, let $p\left(\sigma^{N}\right)$ be the probability distribution over $N$-length sequences of public outcomes induced by $\rho$. Notice that this is a finite vector.

LEMMA 2: For any $\varepsilon>0, N$ there exists a $\gamma>0$ such that in $G_{0}^{N}$ if $\| p\left(\sigma_{1}^{N}, \sigma_{2}^{N}\right)-$ $p\left(\tilde{\sigma}_{1}^{N}, \sigma_{2}^{N}\right) \|<\gamma$ and $\sigma_{2}^{N}$ is an $\varepsilon$-best response by player 2 to $\sigma_{1}^{N}$, then it is a $2 \varepsilon$-best response to $\tilde{\sigma}_{1}^{N} .^{10}$

Proof: We identify type behavior strategies by player 1 that differ only at information sets that are unreachable given that strategy. It is sufficient to show that $p\left(\tilde{\sigma}_{1}^{N}, \sigma_{2}^{N}\right) \rightarrow$ $p\left(\sigma_{1}^{N}, \sigma_{2}^{N}\right)$ implies $\tilde{\sigma}_{1}^{N} \rightarrow \sigma_{1}^{N}$. This in turn will follow if $p\left(\cdot, \sigma_{2}^{N}\right)$ has a continuous inverse. Since the domain of $p\left(\cdot, \sigma_{2}^{N}\right)$ is compact, the image of any closed set in the domain is closed, so it suffices to show that $p\left(\cdot, \sigma_{2}^{N}\right)$ is continuous and $1-1$. Continuity is obvious. To show that $p\left(\cdot, \sigma_{2}^{N}\right)$ is $1-1$ suppose to the contrary that $p\left(\tilde{\sigma}_{1}^{N}, \sigma_{2}^{N}\right)=$ $p\left(\sigma_{1}^{N}, \sigma_{2}^{N}\right)$, but that $\sigma_{1}^{N}, \tilde{\sigma}_{1}^{N}$ are not equivalent. Let $\left(h^{t}, h_{1}^{t}, h_{2}^{t}\right)$ be a triple consisting of a public and private histories (of the same length) possible under $\sigma_{1}^{N}$ such that $\tilde{\sigma}_{1}^{N}\left(h^{t}, h_{1}^{t}\right) \neq \sigma_{1}^{N}\left(h^{t}, h_{1}^{t}\right)$. By Assumption 2 it follows that

$$
\rho\left(\tilde{\sigma}_{1}^{N}\left(h^{t}, h_{1}^{t}\right), \sigma_{2}^{N}\left(h^{t}, h_{2}^{t}\right)\right) \neq \rho\left(\sigma_{1}^{N}\left(h^{t}, h_{1}^{t}\right), \sigma_{2}^{N}\left(h^{t}, h_{2}^{t}\right)\right) .
$$

Since $h$ has positive probability under $\sigma_{1}^{N}$, for some $\tilde{\sigma}_{2}^{N}$, by Assumption 3 it has positive probability under $\sigma_{1}^{N}, \sigma_{2}^{N}$ (and by hypothesis the same probability under $\left.\left(\tilde{\sigma}_{1}^{N}, \sigma_{2}^{N}\right)\right)$. This contradicts $p\left(\tilde{\sigma}_{1}^{N}, \sigma_{2}^{N}\right)=p\left(\sigma_{1}^{N}, \sigma_{2}^{N}\right)$.

Q.E.D.

For a given $\delta_{1}$ let $M\left(K, \jmath_{1}\right)$ be the finite set of probability distributions over types of player 1 that can be generated by Bayesian updating with no more than $K$ observations of player 1's play. ${ }^{11}$

For any fixed $N$, we can partition the set of histories into a sequence of blocks of length $N$. Given a probability distribution $\rho$ over the space $\Omega \times H \times H_{1} \times H_{2}$ of types of

\footnotetext{
${ }^{10}$ The norm \| $\|$ may be taken to be ordinary Euclidean distance, where the probability distribution over a finite set $p(\cdot)$ is viewed as a vector.

${ }^{11}$ Note that, although $\sigma_{2}$ influences the probability of the various elements of $\left.M(K,\lrcorner_{1}\right)$, it does not influence the set itself.
} 
player 1 and public and private infinite histories (in particular, given the distribution induced by a Nash equilibrium of $G^{\infty}$ ) we can then associate every infinite history that has positive probability under $\rho$ with a sequence of $N$-period games of the same form as $G^{N}$, where the prior beliefs in the $N$-period game beginning in period $N(k-1)+1$ are those given by Bayes rule applied to the distribution $\rho$ and to the specified finite history $\left(h^{N(k-1)+1}, h_{1}^{N(k-1)+1}, h_{2}^{N(k-1)+1}\right)$. We will denote this game by $G^{N, k}$, where the dependence on the infinite history $h$ is left implicit.

For any $s_{1}, k$, and for any probability distribution $\mu^{\prime}$ over player 1's types, let $\phi_{1}^{k}\left(h^{N(k-1)+1}, \mu^{\prime}, \jmath_{1}\right)$ be the probability distribution over player 1's strategies in $G_{0}^{N}$ defined by $\phi_{1}^{k}\left(h^{N(k-1)+1}, \mu^{\prime}, \jmath_{1}\right)\left(\sigma_{1}^{N}\right)=\sum_{\omega} \mu^{\prime}(\omega)_{s_{1}}\left(\sigma_{1}^{N} \mid \omega, h^{N(k-1)+1}\right)$. Finally, for a given $\sigma_{2}$, let $\sigma_{2}^{N}\left(h^{t}\right)$ denote the strategy in $G_{0}^{N}$ corresponding to the play of $\sigma_{2}$ in periods $t+1, \ldots, t+N$.

LemMa 3: Fix a Nash equilibrium $\left(s_{1}, \sigma_{2}\right)$ and suppose for some $N$ and $\sigma_{1}^{N}, s_{1}$ is such that type $\omega_{0}$ plays $\sigma_{1}^{N}$ in each $G^{N, k}$. For every $\lambda>0, \gamma>0, K$ there is an $L$ such that if $\mu^{\prime} \in M\left(K, s_{1}\right)$ the probability is less than $\lambda$ that there are more than $L$ stages $G^{N, k}$ with $\left\|p\left(\sigma_{1}^{N}, \sigma_{2}^{N}\left(h^{N(k-1)+1}\right)\right)-p\left(\phi_{1}^{k}\left(h^{N(k-1)+1}, \mu^{\prime}, \jmath_{1}\right), \sigma_{2}^{N}\left(h^{N(k-1)+1}\right)\right)\right\| \geq \gamma$.

Proof: This is a restatement of Theorem 4.1 in Fudenberg and Levine (1992).

Proof of Theorem 1: Fix a Nash equilibrium $\left(s_{1}, \sigma_{2}\right)$. For any number $\eta>0$ we may choose $N, \underline{\delta}_{1}<1, \varepsilon>0, \sigma_{1}^{N}$ so that Lemma 1 is satisfied for the tolerance $\eta / 4$ and hence if player 2 plays an $\varepsilon$-best response to $\sigma_{1}^{N}$ in $G_{0}^{N}$ then the payoff to player 1 in $G_{0}^{N}$ is at least $\bar{\nu}_{1}\left(\delta_{2}\right)-\eta / 4$. The idea is to consider what happens when player 1 of type $\omega_{0}$ repeatedly plays $\sigma_{1}^{N}$ and player 2 plays a best response. Our conclusion follows by demonstrating that if player 1 is more patient than $\underline{\delta}_{1}$, he gets at least $\bar{\nu}_{1}\left(\delta_{2}\right)-\eta$.

To analyze the best response of player 2, we fix an integer $\kappa$ so that $\delta_{2}^{\kappa N}\left(\max u_{2}-\right.$ $\left.\min u_{2}\right)<\varepsilon\left(1-\delta_{2}\right) / 4$. Set $N^{\prime}=\kappa N$. Apply Lemma 2 to $G_{0}^{N^{\prime}}$ where the tolerance is $\varepsilon\left(1-\delta_{2}\right) / 2$ to find a value for $\gamma$. Apply Lemma 3 to blocks $G^{N^{\prime}}$ using this same value of $\gamma$, and choosing $\lambda$ such that

$$
\left(1-(1-\lambda)^{\kappa}\right)\left(\max u_{1}-\min u_{1}\right)<\frac{\eta}{4} .
$$

We refer to blocks $G^{N^{\prime}, k}$ in which

$$
\begin{aligned}
& \|_{p}\left(\sigma_{1}^{N^{\prime}}, \sigma_{2}^{N^{\prime}}\left(h^{N^{\prime}(k-1)+1}\right)\right) \\
& \quad-p\left(\phi_{1}^{k}\left(h^{N^{\prime}(k-1)+1}, \mu^{\prime}, \jmath_{1}\right), \sigma_{2}^{N^{\prime}}\left(h^{N^{\prime}(k-1)+1}\right)\right) \|_{\geq \gamma}
\end{aligned}
$$

as anomalous.

Strategy $\sigma_{2}$ is at worst an $\varepsilon\left(1-\delta_{2}\right) / 2$ best response to

$$
\phi_{1}^{k}\left(h^{N^{\prime}(k-1)+1}, \mu\left(h^{N^{\prime}(k-1)+1}\right), \jmath_{1}\right)
$$

in $G^{N^{\prime}, k}$ where $\mu\left(h^{N^{\prime}(k-1)+1}\right)$ denotes 2's posterior beliefs given $h^{N^{\prime}(k-1)+1}$ (this is the case since $\left.\delta_{2}^{\kappa N}\left(\max u_{2}-\min u_{2}\right)<\varepsilon\left(1-\delta_{2}\right) / 4\right)$. By Lemma 2 player 2 is playing an $\varepsilon\left(1-\delta_{2}\right)$ best response to the $\kappa$-fold repetition of $\sigma_{1}^{N}$ except in the anomalous blocks. 
Consequently, except in anomalous blocks, player 2 is playing an $\varepsilon$-best response to $\sigma_{1}^{N}$ in the first $N$ periods of each $N^{\prime}$ block. Lemma 1 then shows that player 1 gets at least $\bar{\nu}_{1}\left(\delta_{2}\right)-\eta / 2$ in the first $N$ periods of each nonanomalous block. Furthermore, with probability at least $1-\lambda$ there are at most $L$ anomalous blocks.

Now consider the infinitely repeated game beginning in any period $k N+1,1 \leq k \leq \kappa-$ 1. This is identical to the game that begins in period 1 , except that the prior of player 2 may have changed. We may again organize this game into blocks of length $N^{\prime}$ and, since Lemma 3 applies to all priors reachable during periods up to $N^{\prime}$, the previous argument shows that for every $k$ there are at most $L$ anomalous blocks with probability at least $1-\lambda$. Thus with probability at least $(1-\lambda)^{\kappa}$ in the overall game there are at most $\kappa L$ anomalous blocks. The payoff of player 1 is therefore at least

$$
\delta_{1}^{\kappa L \kappa N} \bar{\nu}_{1}\left(\delta_{2}\right)-3 \eta / 4+\left(1-\delta_{1}^{\kappa L \kappa N}\right) \min u_{1} .
$$

Letting $\delta_{1} \rightarrow 1$ gives the desired result.

Q.E.D.

\section{A PATIENT PLAYER 2}

Our second Theorem shows that as $\delta_{2} \rightarrow 1$ player 1 can approximately obtain the maximum feasible payoff that gives player 2 his pure strategy minmax payoff. Note that this bound is derived by taking a particular order of limits. First, we derive a lower bound on player 1's payoff as $\delta_{1} \rightarrow 1$, and then we ask how this bound behaves as $\delta_{2} \rightarrow 1$. Let $V^{*}$ denote the convex hull of feasible payoffs that are at least as great as the pure strategy minmax. By $V_{1}^{*}$ we denote the projection of $V^{*}$ onto the payoffs of player 1 .

THEOREM 2: Suppose Assumptions 1-4 hold. Then $\liminf {\delta_{2} \rightarrow 1}_{1} \liminf _{\delta_{1} \rightarrow 1} E_{1}\left(\delta_{1}, \delta_{2}\right) \geq$ $\max V_{1}^{*}$.

It is worth noting that if we allowed types of player 2, this result would remain valid, and the proof (and Theorem 1 from which it follows) would involve only notational changes. However, we cannot turn the Theorem around and use the fact that there are types of player 2 to find a bound on his payoffs similar to that for player 1: The validity of Theorem 2 depends crucially on the order of limits.

Note also that the definition of $V^{*}$ makes use of the pure strategy minmax. As was argued in Section 2, we allow player 1 to establish a reputation only for a pure strategy, since allowing him to establish a reputation for mixed strategy punishments would require the existence of a continuum of types that would make the notation significantly heavier. If we allowed a reputation for mixed strategies, we could use the usual feasible individually rational set, and replace the inequality in Theorem 2 with an equality.

The proof of Theorem 2 is an immediate consequence of Theorem 1 and the following Lemma.

LEMMA 4: For any $\nu=\left(\nu_{1}, \nu_{2}\right)$ with $\nu \in$ int $V^{*}$ there is a $\bar{\delta}_{2}$ such that, for $\delta_{2}>\bar{\delta}_{2}$, $\nu_{1} \in V_{1}\left(\delta_{2}\right)$.

REMARK: We should emphasize that this Lemma concerns the complete information game, where reputation plays no role. The Lemma is thus more closely related to the 


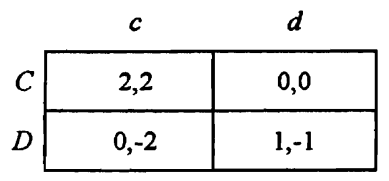

FIGURE 2

literature on repeated games than to that on reputation effects, and indeed our proof uses "review strategies" of the sort introduced in Radner's $(1981,1985)$ study of repeated agency games, and subsequently used in a number of papers on the folk Theorem in repeated games. Despite this close link to the repeated game literature, the Lemma we need does not seem to be a direct consequence of previous work, so we give a complete proof in the Appendix.

\section{EXAMPLE}

In this Section we give an example that shows that the conclusions of Theorems 1 and 2 fail with perfectly observable actions even if the equilibrium concept is strengthened to perfect Bayesian equilibrium. ${ }^{12}$

Suppose that the following stage game (see Figure 2) is played repeatedly with perfect observability. Player 1 is the row player and player 2 the column player. Notice that the Stackelberg payoff here is 2 , with player 1 playing $C$ and player 2 playing $c$. Suppose that there are two types of patient player: type $\omega_{0}$ is the normal type and type $\omega_{1}$ always plays $C$; note that there are no perverse types. The prior probability of the commitment type is $\mu_{1}=.001$. For simplicity we assume that there is a public randomization device.

We claim that for all $\delta_{1} \geq \delta_{2} \geq .99$ there is a perfect Bayesian equilibrium ${ }^{13}$ in which type $\omega_{0}$ 's payoff is no more than 1 ; in particular this is the case if $\delta_{1}=\delta_{2} \geq .99 .{ }^{14}$ The equilibrium strategies we describe below can be adapted to support any payoff of type $\omega_{0}$ of player 1 strictly above his minmax payoff of $2 / 3$, if $\delta_{1}>\delta_{2}>\bar{\delta}$ for some $\bar{\delta}<1$.

Notice that if player 1 ever plays $D$, player 2 learns for certain that 1 is the normal type; at this point every equilibrium of the game where 1 is known to be normal (the "complete-information game") is a continuation equilibrium of the original game. For this reason, it is useful to consider the perfect equilibria of the complete-information repeated game. First, there are three static equilibria: $(2,2)$ and $(1,-1)$ are pure strategy

\footnotetext{
${ }^{12}$ Moreover this example is robust to small changes in payoffs, and player 1 cannot obtain the Stackelberg payoff by commitment to a mixed strategy. We thank a referee for finding an error in a previous example, and for encouraging us to find a robust example.

${ }^{13}$ Although sequential equilibrium has not been defined for infinite games, in the finitely repeated versions of the two-types example considered here the outcomes of perfect Bayesian equilibria (PBE) and sequential equilibria coincide (Fudenberg and Tirole (1991)). We use the simpler PBE concept because its conditions on beliefs are easier to check.

${ }^{14}$ Cripps and Thomas (1993) give a related example for the case where both players have the same discount factor.
} 
equilibria, and there is a mixed strategy equilibrium in which player 1 plays $C$ with probability $1 / 3$ and player 2 plays $c$ with probability $1 / 3$; the payoff vector to this mixed strategy equilibrium is $(2 / 3,-2 / 3)$. Naturally, the infinite repetition of any of these static equilibria is a subgame-perfect equilibrium of the repeated game. Moreover, any payoff vector $\left(u_{1}, u_{2}\right)$ in the convex hull of the pure strategy payoffs that satisfies $\left(u_{1}, u_{2}\right) \geq(.687,-.646)$ can also be obtained in a perfect equilibrium. These payoffs can be achieved by strategies that specify a repeated public randomization yielding $\left(u_{1}, u_{2}\right)$ each period along the equilibrium path, with play switching to the static mixed strategy equilibrium if any player deviates. ${ }^{15}$

Finally, when the two players have different discount factors, there are socially feasible payoffs that are not in the convex hull of pure strategy payoffs. Call a sequence of expected present values $\left(\nu_{1}(t), \nu_{2}(t)\right)$ socially feasible if there is a strategy profile that gives rise to this sequence. If in each period $\left(\nu_{1}(t), \nu_{2}(t)\right) \geq(.687,-.646)$, then each payoff vector in the sequence is a perfect equilibrium payoff: again the punishment is reversion to the static mixed strategy equilibrium. A particular example of such payoffs are any payoffs of the form $\left(\delta_{1}^{\tau} u_{1}, \delta_{2}^{\tau} u_{2}\right) \geq(.687,0)$ where $\left(u_{1}, u_{2}\right)$ is an element of the convex hull of pure strategy payoffs. (Note that payoffs of this form need not themselves be a convex combination of pure strategy payoffs.) Such payoff sequences can be realized by playing $(C, d)$ starting in period 1 and for $\tau$ periods, and then switching to the stationary equilibrium $\left(u_{1}, u_{2}\right)$. That this nonstationary sequence is an equilibrium follows from:

$$
\begin{aligned}
\left(\nu_{1}(t), \nu_{2}(t)\right) & =\left(\delta_{1}^{\tau-t-1} u_{1}, \delta_{2}^{\tau-t-1} u_{2}\right) \\
& \geq\left(\delta_{1}^{\tau} u_{1}, \delta_{2}^{\tau} u_{2}\right) \geq(.687,0), \quad t \leq \tau+1
\end{aligned}
$$

We will now describe a perfect Bayesian equilibrium of the incomplete information game in which type $\omega_{0}$ 's equilibrium payoff is exactly equal to 1 . We will describe the equilibrium through its equilibrium path and continuation equilibria that arise in response to deviations.

The equilibrium path: Along the equilibrium path player 1 always plays $C$ while a $(.5, .5)$ public randomization determines whether player 2 chooses $d$ or $c$. This implies that both players get a present value of 1 . No information about player 1's type is revealed on the equilibrium path, so player 2 does not update his beliefs.

Deviations from the equilibrium path: If both players deviate simultaneously both deviations are ignored. ${ }^{16}$ If either player is the only deviator in a period in which player 2 is supposed to play $c$, the deviation is ignored. This is consistent with equilibrium, since such deviations do not yield a short term gain. If player 1 is the only deviator in a period

\footnotetext{
${ }^{15}$ The bound on player 1's payoff is derived as follows. If the public randomization prescribes the play of $(D, c)$, player 1 obtains $2\left(1-\delta_{1}\right)+(2 / 3) \delta_{1}$ from deviating, and $0+\delta_{1} \nu_{1}$ from conforming, where $\nu_{1}$ is player 1's equilibrium payoff; conforming is better than deviating if $\nu_{1}$ is greater than $.68 / .99<.687$. Similar calculations show that this bound on 1's equilibrium payoff implies that player 1 will not want to deviate when any of the other three pure strategy profiles are supposed to be played. The computations for player 2 are analogous.

${ }^{16}$ Play following simultaneous deviations is always irrelevant to the determination of equilibrium.
} 
where 2 plays $d$, player 1 is revealed to be a normal type, and play switches to the repeated mixed strategy equilibrium of the complete information game. In this case, the deviation yields $\left(1-\delta_{1}\right)+\delta_{1} 2 / 3$ which is less than the equilibrium value of 1 for any $\delta_{1}$. Finally, if player 2 is the only deviator in a period in which 2 is supposed to play $d$, no information is revealed about player 1's type, and play switches to a perfect Bayesian equilibrium of the incomplete information game that we refer to as the player 2 punishment equilibrium. We verify below that the expected present value to player 2 in this punishment equilibrium is no greater than .81 . Since $1 \geq\left(1-\delta_{2}\right) \cdot 2+.81 \delta_{2}$, this will imply that player 2 has no incentive to deviate from the equilibrium path.

Player 2 punishment equilibrium: Along the equilibrium path, play has two phases. The first phase lasts at most $\tau$ periods, where $\delta_{2}^{\tau} \geq 0.4 \geq \delta_{2}^{\tau+1}$. In this phase, the normal type of player 1 mixes between $C, D$ as described below, while player 2 chooses $d$. The first phase ends when either time runs out (i.e., after $\tau$ periods) or when player 1 plays $D$. If time runs out play switches to the cooperative equilibrium in which regardless of observed play both types of player 1 play $C$ and player 2 plays $c$. This is clearly a perfect Bayesian equilibrium. If player 1 plays $D$ in the $t$ th period of the punishment equilibrium, he reveals his type and in the following period play switches to an equilibrium of the complete information game in which $(C, d)$ is played up until and including period $\tau$, and whose present value payoffs are $\left(\delta_{1}^{\tau-t} u_{1}(t), \delta_{2}^{\tau-t} u_{2}(t)\right)$, where

$$
\left(u_{1}(t), u_{2}(t)\right)=\left(2-\frac{1-\delta_{1}}{\delta_{1}^{\tau-t+1}}, 2-\frac{1-\delta_{1}}{\delta_{1}^{\tau-t+1}}\right) .
$$

It can easily be checked that the vector $\left(u_{1}(t), u_{2}(t)\right)$ is socially feasible for all $1 \leq t \leq \tau$, and that $\left(\delta_{1}^{\tau-t} u_{1}(t), \delta_{2}^{\tau-t} u_{2}(t)\right) \geq\left(\delta_{1}^{\tau-1} u_{1}(1), \delta_{2}^{\tau-1} u_{2}(1)\right) \geq(.79, .79)$. So from our earlier analysis these are indeed perfect equilibrium payoffs of the complete information game.

Notice that the payoffs to player 1 following play of $D$ are calibrated so that he is exactly indifferent between $C$ and $D$, implying that he is willing to use a mixed strategy during the first phase, as we have specified. The exact mixing probabilities player 1 uses will be determined, as usual, by the consideration of his opponent's incentives, to which we now turn.

If player 2 deviates in a period when the realization of player 1's strategy is $C$, the deviation is ignored. If player 2 deviates in the first phase and the realization of player 1's action is $D$, then play switches to the static mixed strategy equilibrium of the complete information game where player 1 is known to be the normal type. ${ }^{17}$

To show that the deviation is not optimal for player 2, we must now specify the randomization probabilities for player 1 during the first phase of the punishment equilibrium, and the corresponding beliefs for player 2 conditional on observing a string of $C$ played by player 1 in all $t \leq \tau$ during the first phase. These in turn will determine player 2's expected payoff from conforming to the specified strategies at such histories, which in turn will determine whether player 2 can gain by deviating.

Denote by $\mu_{t}$ player 2's subjective probability that player 1 is a commitment type in the $t$ th period of the first phase. Let $p=1-6\left(1-\delta_{2}\right)$. In the $t$ th period of this phase, type $\omega_{0}$ chooses $C$ with probability $p_{t}=\left(p-\mu_{t}\right) /\left(1-\mu_{t}\right)$, so that the overall probability

\footnotetext{
${ }^{17}$ Note that playing $D$ reveals that player 1 is the normal type.
} 
that player 1 chooses $C$ equals $p$. The beliefs of player 2 in the first phase are then given by $\mu_{t}=\mu_{0} / p^{t-1}$. Since $\tau \leq \ln (.4) / \ln \left(\delta_{2}\right)$ we have

$$
\begin{aligned}
\mu_{t} & \leq \frac{\mu_{0}}{p^{\tau}} \\
& \leq \frac{\mu_{0}}{\left(1-6\left(1-\delta_{2}\right)\right)^{\left(\ln (.4) / \ln \left(\delta_{2}\right)\right)}} \\
& \leq \frac{\mu_{0}}{(1-6(1-.99))^{(\ln (.4) / \ln (.99))}}=.282 \\
& <.94=1-6(1-.99) \leq 1-6\left(1-\delta_{2}\right)=p
\end{aligned}
$$

for $\delta_{2}>.99{ }^{18}$ Therefore, since $p>\mu_{t}, 1 \leq t \leq \tau, p_{t}$ is greater than 0 .

Let $V_{2}^{t}$ denote player 2's expected payoff in period $t$ of the first phase of the punishment equilibrium. To show that player 2 loses by deviating in the first phase of the punishment equilibrium, we first provide a lower bound for $V_{2}^{t}, 1 \leq t \leq \tau$.

After the first $\tau$ periods of the punishment equilibrium, the continuation payoff of player 2 is either 2 , if time ran out, or $2-\left(1-\delta_{1}\right) /\left(\delta_{1}^{\tau-t+1}\right)$, if in the first phase the realization of player 1's strategy was $D$ in period $t$, where $1 \leq t \leq \tau$. It is therefore easy to see that $V_{2}^{t}$ is bounded below by

$$
V_{2}^{t} \geq-\left(1-\delta_{2}\right)+2 \delta_{2}^{\tau+1-t}-\frac{1-\delta_{1}}{\delta_{1}^{\tau+1-t}} \cdot \delta_{2}^{\tau+1-t} \geq .78
$$

We claim that player 2 has no incentive to deviate in the punishment equilibrium if

$$
\begin{gathered}
2\left(1-\delta_{2}\right)+\delta_{2}\left[p V_{2}^{t+1}+(1-p) \delta_{2}(-2 / 3)\right] \\
\leq(1-p)\left(1-\delta_{2}\right)(-1)+\delta_{2} V_{2}^{t+1} .
\end{gathered}
$$

To see this, note that the right-hand side of the inequality is player 2's expected payoff from conforming to $d$, and that the left-hand side is an overestimate of his payoff from playing $c$, since it calculates player 2's current-period payoff as if player 1's realized action were sure to be $C$. Note next that this inequality is equivalent to

$$
\begin{aligned}
& 2\left(1-\delta_{2}\right)+\delta_{2}\left(6\left(1-\delta_{2}\right)\right) \delta_{2}(-2 / 3) \\
& \quad \leq 6\left(1-\delta_{2}\right)\left(1-\delta_{2}\right)(-1)+\delta_{2} 6\left(1-\delta_{2}\right) V_{2}^{t+1} .
\end{aligned}
$$

Since $V_{2}^{t} \geq .78$ this inequality holds if

$$
2+\delta_{2} \cdot 6 \cdot \delta_{2}(-2 / 3) \leq 6\left(1-\delta_{2}\right)(-1)+\delta_{2} \cdot 6 \cdot 0.78
$$

which is satisfied for $\delta_{2} \geq .99$. This shows that player 2 has no profitable deviation from the first phase of his punishment equilibrium.

Finally note that $.81 \geq 2 \delta_{2}^{\tau} \geq V_{2}^{1}$, so that player 2's expected payoff in the initial period of the punishment equilibrium is indeed no greater than .81 . As was remarked above, this shows that player 2 cannot gain by deviating along the equilibrium path.

${ }^{18}$ Note that

$$
\frac{\mu_{0}}{\left(1-6\left(1-\delta_{2}\right)\right)^{\left(\ln (.4) / \ln \left(\delta_{2}\right)\right)}} \rightarrow \frac{\mu_{0}}{.4^{6}}=.244 \quad \text { as } \quad \delta_{2} \rightarrow 1 .
$$


Dept. of Economics, Universidad Carlos III de Madrid, Calle Madrid 126, Getafe (Madrid) 28903, Spain,

Dept. of Economics, Harvard University, Cambridge, MA 02138, U.S.A.,

Dept. of Economics, University of California-Los Angeles, Los Angeles, CA 90024, U.S.A.,

and
Dept. of Economics, Northwestern University, Evanston, IL 60201, U.S.A.

Manuscript received December, 1993; final revision received June, 1995.

\begin{abstract}
APPENDIX
Proof of Lemma 4: Given $\nu=\left(\nu_{1}, \nu_{2}\right)$ with $\nu \in \operatorname{int} V^{*}$ let $\vec{a}_{1}=\left(\vec{a}_{1}^{1}, \ldots, \vec{a}_{1}^{N}\right)$ be a sequence of actions by player 1 such that there is a sequence of actions for player 2 such that in $G_{0}^{N}$ the expected average payoff of player 1 is larger than $\nu_{1}-\varepsilon / 2$ and the expected average payoff of player 2 is larger than $\nu_{2}$. (Clearly for $N$ sufficiently large such a sequence exists.)

Consider the $K M N$-fold repeated game $G_{0}^{K M N}$ in which player 2 has the time average payoff as a payoff function. Again we can partition $G_{0}^{K M N}$ into blocks of length $M N, G_{0}^{M N, k}, k=1, \ldots, K$. Denote by $\overrightarrow{\vec{a}}_{1}=\left(\vec{a}_{1}, \ldots, \vec{a}_{1}\right)$ the sequence of $M$ repetitions of $\vec{a}_{1}$. Let $\underline{a}_{1}$ be a (pure) action of player 1 that minmaxes the payoff of player 2 in pure strategies. Further, let $u_{1}^{k}$ denote player 1 's average payoff in $G_{0}^{M N, k}$.

Let $\hat{\sigma}_{1}$ be the following strategy: in $G_{0}^{M N, 1}$, player 1 chooses $\overrightarrow{\vec{a}}_{1}$. In $G_{0}^{M N, 2}$ if $\nu_{1}-u_{1}^{1}-\varepsilon / 2<\eta$, then player 1 again chooses $\overrightarrow{\vec{a}}_{1}$ and so on. If for any $k, \nu_{1}-u_{1}^{k}-\varepsilon / 2>\eta$, then player 1 plays action $\underline{a}_{1}$ for the next $P$ repetitions of $G_{0}^{M N, k}$.

CLAIM: Given $\varepsilon>0$ there are numbers $\eta, K, N, M$, and $P$ with $P / K<\varepsilon$ such that for any (time average) best response $\hat{\sigma}_{2}$ to $\hat{\sigma}_{1}$ the following is satisfied:

(i) if player 1 chooses $\overrightarrow{\vec{a}}_{1}$ in $G_{0}^{M N, k}, k=1, \ldots, K-P$, then $\operatorname{Pr}\left(\nu_{1}-u_{1}^{k}<\varepsilon\right)>1-\varepsilon$.

(ii) the fraction of stages $k$ in which player 1 uses his punishment strategy is smaller than $\varepsilon$ with probability $(1-\varepsilon)$.
\end{abstract}

Assuming for the moment the truth of the claim, a straightforward upper hemi-continuity argument shows that the claim remains true if $\hat{\sigma}_{2}$ is a discounted best response for $\delta_{2}$ sufficiently close to 1 . Moreover, the claim implies that the average payoff to player 1 in $G_{0}^{K M N}$ is greater than $\nu_{1}-B \varepsilon$ where $B$ is a positive constant independent of $\varepsilon$. This is the desired result.

To demonstrate the validity of the claim, first choose $\eta<\varepsilon^{2} /(\beta+1)$, where $\beta$ is a fixed constant whose computation is described below.

Denote by $E\left[u_{1}^{k} \mid h^{(k-1) M N+1}\right]$ the expected payoff of player 1 in $G_{0}^{M N, k}$ given the history $h^{(k-1) M N+1}$. Given $K$ and $\eta$ we can choose $M$ sufficiently large, so that:

(a) if $\nu_{1}-E\left[u_{1}^{k} \mid h^{(k-1) M N+1}\right]-\varepsilon / 2>2 \eta$, then punishment occurs with probability greater than $(1-\eta)$ in $G_{0}^{M N, k}$;

(b) if $\nu_{1}-E\left[u_{1}^{k} \mid h^{(k-1) M N+1}\right]-\varepsilon / 2<\eta$ for all $k$ and for all histories $h^{(k-1) M N+1}$ such that player 1 chooses $\overrightarrow{\vec{a}}_{1}$ in $G_{0}^{M N, k}$, then the probability that no punishment occurs in any $G_{0}^{M N, k}$ is larger than $(1-\eta)$.

Note that the utility loss from a punishment is bounded below by $\left(\nu_{2}-\underline{u}_{2}\right) P,\left(\nu_{2}-\underline{u}_{2}>0\right)$, whereas the gain from a deviation is bounded above by $\bar{u}_{2}-\underline{u}_{2}$, where $\bar{u}_{2}$ is the largest attainable payoff for player 2 in the stage game. Thus for appropriate choice of $P$ we have

$$
\nu_{1}-E\left[u_{1}^{k} \mid h^{(k-1) M N+1}\right]-\varepsilon / 2<2 \eta
$$

in all of the first $K-P$ stages for any best response of player 2 and for all histories $h^{(k-1) M N+1}$ such that player 1 chooses $\overrightarrow{\vec{a}}_{1}$ in $G_{0}^{M N, k}$. Hence part (i) of the claim follows. 
Now we establish part (ii) of the claim. Suppose to the contrary that player 2's (optimal) strategy triggers a punishment in more than an $\varepsilon$ fraction of the first $K-P$ stages with probability greater than $\varepsilon$. We claim that this implies that a profitable deviation exists. Suppose player 2 deviates so that $\nu_{1}-E\left[u_{1}^{k} \mid h^{(k-1) M N+1}\right]-\varepsilon / 2<\eta$ for all $k=1, \ldots, K-P$ and for all histories $h^{(k-1) M N+1}$ such that player 1 chooses $\overrightarrow{\vec{a}}_{1}$ in $G_{0}^{M N, k}$. Since inequality (A1) has to be satisfied for all $k=1, \ldots, K$ $-P$, this deviation can be chosen so that the loss in every stage is bounded above by $\eta\left(\max u_{2}-\right.$ $\min u_{2}$ ). Note that (after the deviation) the probability that a punishment occurs in any stage is smaller than $\eta$. Thus player 2 also improves his average payoff over the first $K-P$ stages by at least $\left(\varepsilon^{2}-\eta\right)\left(\nu_{2}-\underline{u}_{2}\right) \cdot P$ by reducing the probability of punishment. Consequently, if we choose

$$
\beta=\frac{\left(\max u_{2}-\min u_{2}\right) K}{\left(\nu_{2}-\underline{u}_{2}\right) \cdot P},
$$

player 2 gains from the deviation and part (ii) of the claim follows.

Q.E.D.

\section{REFERENCES}

AOYAGI, M. (1994): "Reputation and Dynamic Stackelberg Leadership in Infinitely Repeated Games," mimeo.

Celentani, M. (1996): "Reputation with Observable Actions," Economic Theory, forthcoming.

Celentani, M., and W. Pesendorfer (1996): "Reputation in Dynamic Games," Journal of Economic Theory, forthcoming.

Celentani, M., D. Fudenberg, D. K. Levine, and W. Pesendorfer (1993): "Maintaining a Reputation Against a Patient Opponent," CMSEMS Discussion Paper No. 1075, Northwestern University.

CripPs, M., K. Schmidt, ANd J. P. Thomas (1996): "Reputation in Perturbed Repeated Games," Journal of Economic Theory, forthcoming.

CRIPPS, M., AND J. P. Thomas (1993): "Reputation and Perfection in a Repeated Common Interest Game," mimeo.

(1994): "Reputation and Equilibrium Selection in Two-Person Repeated Games without Discounting," mimeo.

FudENBERG, D., AND D. M. KREPS (1987): “Reputation and Simultaneous Opponents," Review of Economic Studies, 54, 541-568.

Fudenberg, D., AND D. K. Levine (1983): "Subgame Perfect Equilibria of Finite and Infinite Horizon Games," Journal of Economic Theory, 31, 251-268.

- (1989): "Reputation and Equilibrium Selection in Games with a Single Patient Player," Econometrica, 57, 759-778.

- (1992): "Maintaining a Reputation when Strategies are Imperfectly Observed," Review of Economic Studies, 59, 561-579.

FudenBerg, D., AND J. TiRole (1991): “Perfect Bayesian Equilibrium and Sequential Equilibrium," Journal of Economic Theory, 53, 236-260.

KREPS, D. M., AND R. WILSON (1982): "Reputation and Imperfect Information," Journal of Economic Theory, 27, 253-279.

Milgrom, P., AND J. RoBerTs (1982): "Predation, Reputation and Entry Deterrence," Journal of Economic Theory, 27, 280-312.

RADNER, R. (1981): “Monitoring Cooperative Agreements in a Repeated Principal-Agent Relationship," Econometrica, 49, 1127-1148.

- (1985): "Repeated Principal Agent Games with Discounting," Econometrica, 53, 1173-1198.

SCHMIDT, K. (1993): "Reputation and Equilibrium Characterization in Repeated Games with Conflicting Interests," Econometrica, 61, 325-351. 